\title{
Novel Radio Resource Management Scheme with Low Complexity for Multiple Antenna Wireless Network System
}

\author{
Jian Xu, Rong Ran, DongKu Kim, and Jong-Soo Seo \\ Department of Electrical and Electronic Engineering, Yonsei University, Shinchon Dong, \\ Seodaemoon-Ku, Seoul, 120-749, Korea \\ jianxu@yonsei.ac.kr
}

\begin{abstract}
Multiple-input multiple-output (MIMO) antennas can be combined with orthogonal frequency division multiplexing (OFDM) to increase system throughput by spatial multiplexing. That is the requirement of high speed future wireless networks such as WLANs and WMANs. This paper investigates the radio resource management problem of MIMO-OFDM based wireless network systems in order to maximize the total system throughput subject to the total power and proportional rate constraints of each user. A low-complexity algorithm that separates subcarrier allocation and power allocation is proposed. Simulation results show that the proposed resource allocation algorithm can improve the throughput and also it can make the throughput be distributed more fairly among users than some other schemes.
\end{abstract}

Keywords: WLANs, Radio Resource Management, Multiple Antenna, OFDM.

\section{Introduction}

Implementation of high-data-rate wireless local area network (WLAN) has been a major focus of research in recent years. Multiple-input multiple-output (MIMO) schemes [1] and [2] and orthogonal frequency division multiplexing (OFDM) [3] can be combined to operate at the high-throughput (HT) mode, or the diversity mode, or the combination of both in fading environments [4]. Such systems could achieve high spectral efficiency and/or a large coverage area that are critical for future-generation wireless local area networks.

Common open-loop linear detection schemes include the zero-forcing $(\mathrm{ZF})$ and minimum mean-square error (MMSE) schemes [5] and [6]. A large condition number (i.e., the maximum-to-minimum-singular-value ratio, MMSVR) of the channel state information (CSI) matrix implies a high noise enhancement. Thus, MMSVR could be a convenient and effective metric to characterize the performance of different MIMO configurations. The importance and effectiveness of the eigenvalue distribution on MIMO system capacity and the overall system performance have been well recognized [7]-[10]. The eigenvalue analysis for MIMO-OFDM systems can be used to reduce the overall system complexity [11] and [12]. In this paper, the MMSVR is used for the subcarrier allocation, in this way the allocation complexity can be reduced and also the detection performance in the receiver side could be improved. 
In recent years, however, many dynamic subcarrier, power allocation algorithms for single input single output (SISO) OFDM systems have been developed to find the solution of maximizing system throughput or minimizing the overall transmit power [13]-[15]. These suboptimal algorithms have good performances, but cannot be applied to the MIMO-OFDM system. Few researches [16]-[17] have been done for the dynamic subcarrier, power and bit allocation in the MIMO-OFDM system.

In this paper, we investigate the subcarrier and power allocation problems for MIMO-OFDM based wireless network system. We concentrate more on throughput fairness among the users. Our objective is to maximize the total throughput of the system subject to the total power and proportional rate constraints of each user. By dealing subcarrier and power allocation issues separately, we can simplify the resource allocation problem. We proposed a subcarrier allocation algorithm by dividing the users into groups and the MMSVR is treated as an important criterion to pick up the subcarriers for each user.

This paper is organized as follows. Section 2 introduces the MIMO-OFDM system model and presents the optimization objective function. In Section 3, the proposed radio resource management algorithm is described. Simulation results are illustrated in Section 4 and conclusions are drawn in Section 5.

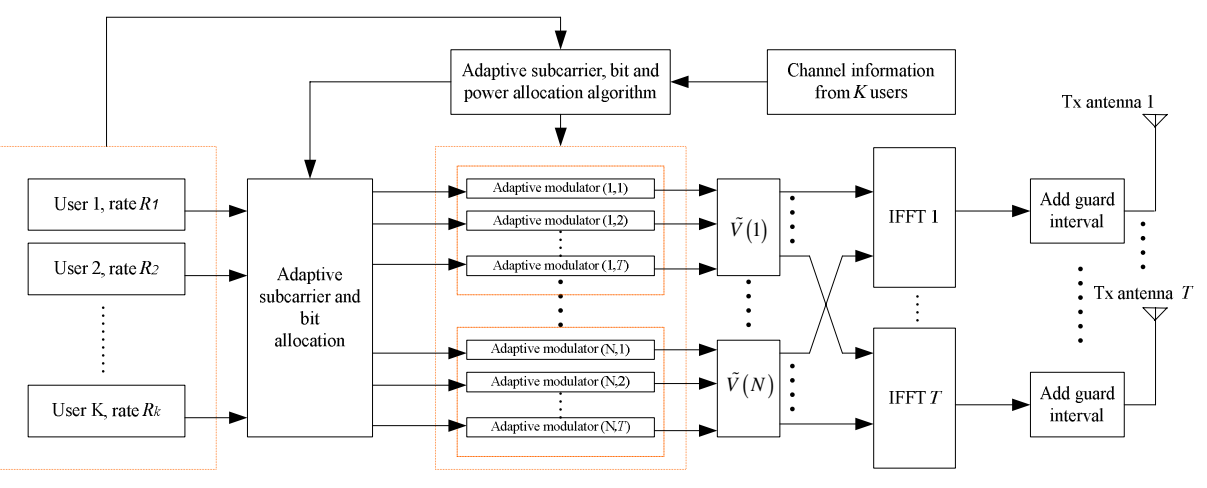

Fig. 1. Block diagram of MIMO-OFDMA system in the downlink

\section{System Model and Problem Formulation}

\subsection{System Model}

The block diagram of adaptive MIMO OFDMA system is shown in Fig.1. In this paper, it is assumed that in the base station the channel state information of each couple of transmit and receiver antennas is sent to the subcarrier and power algorithm block through the feedback channels. The resource allocation information is forwarded to the MIMO-OFDM transmitter. The transmitter then selects the allocated number of bits from different users to form OFDMA symbols and transmits via all the transmit antennas. The spatial multiplexing mode of MIMO is considered. The resource allocation scheme is updated as soon as the channel information is collected and also the subcarrier and bit allocation information is sent to each user for detecting. 


\subsection{Problem Formulation}

Throughout this paper, let the number of transmit antennas be $T$ and the number of receiver antennas be $R$ for all users. Denote the number of users as $K$ and the number of subcarriers as $N$. Assume that the base station has total transmit power constraint $Q$. The objective is to maximize the total system throughput and consider the fairness between the users with the total power constraint. We use the equally weighted sum throughput as the objective function. We also include a set of nonlinear constraints so that we can control the throughput ratios among users.

We formulate the following system throughput optimization problem to determine the subcarrier allocation and power distribution:

$$
\begin{array}{cl}
\max C=\frac{W}{N} \sum_{k=1}^{K} \sum_{n=1}^{N} \rho_{k, n}\left(\sum_{i=1}^{M_{k n}} \log \left(1+\frac{\lambda_{k n}^{(i)} q_{k, n}}{N_{0}}\right)\right) \\
\text { subject to: } \quad \sum_{k=1}^{K} \sum_{n=1}^{N} q_{k, n} \leq Q . \\
& q_{k, n} \geq 0 \text { for all } k, n \\
& \rho_{k, n}=\{0,1\} \text { for all } k, n \\
& \sum_{k=1}^{K} \rho_{k, n}=1 \text { for all } n \\
& R_{1}: R_{2}: \ldots: R_{K}=\gamma_{1}: \gamma_{2}: \ldots: \gamma_{K}
\end{array}
$$

where $K$ is the total number of users; $N$ is the total number of subcarriers; $Q$ is the total available power; $W$ is the system bandwidth; $q_{k, n}$ is the power allocated for user $k$ in the subcarrier $n ; M_{k n}$ is the rank of $H_{k n}$ which denotes the channel gain matrix $(R \times T)$ on subcarrier $n$ for user $k$ and $\left\{\lambda_{k n}^{(i)}\right\}_{i=1: M_{k n}}$ are the eigenvalues of $H_{k n} H_{k n}^{\dagger} ; \rho_{k, n}$ can only be the value of 1 or 0 indicating whether subcarrier $n$ is used by user $k$ or not. $N_{0}$ is the noise power in the frequency band of one subcarrier.

The throughput for user $k$, denoted as $R_{k}$, is defined as

$$
R_{k}=\frac{W}{N} \sum_{n=1}^{N} \rho_{k, n}\left(\sum_{i=1}^{M_{k n}} \log \left(1+\frac{\lambda^{(i)}{ }_{k n} q_{k, n}}{N_{0}}\right)\right)
$$

and $\left\{\gamma_{i}\right\}_{i=1}^{K}$ is a set of predetermined values which are used to ensure proportional fairness among users. 


\section{Proposed Radio Resource Management Scheme}

Ideally, subcarriers and power should be allocated jointly to achieve the optimal solution in (1). However, this poses a prohibitive computational burden at the access point or base station in order to reach the optimal allocation. Furthermore, the access point has to rapidly compute the optimal subcarrier and power allocation as the wireless channel changes. Hence, low-complexity suboptimal algorithms are preferred for cost-effective and delay-sensitive implementations. Separating the subcarrier and power allocation is a way to reduce the complexity, because the number of variables in the objective function is almost reduced by half.

Before we describe the proposed suboptimal resource allocation algorithm, we firstly show the mathematical expression of MMSVR. Let $\eta_{k, n}=\sigma_{k, n}^{\max } / \sigma_{k, n}^{\min }$ denotes the maximum to minimum singular value ratio for user $k$ on subcarrier $n . \sigma_{k, n}^{\max }$ and $\sigma_{k, n}^{\min }$ are the maximum singular value and the minimum singular value of $H_{k n}$, respectively.

A large $\eta_{k, n}$ value could arise either because $\sigma_{k, n}^{\min }$ is small or because $\sigma_{k, n}^{\max }$ is large. From simulation results, it is found that the latter is unlikely, thus $\eta_{k, n}$ is a good indicator of noise enhancement, and if $\eta_{k, n} \gg 1$, we can conclude that the channel is ill-conditioned for the $n$th sub-carrier. In the following step, this point will be adopt as the important rule to allocate the subcarrier, which can avoid using the illconditioned subcarrier so that the noise enhancement could be reduced.

The steps of the proposed suboptimal algorithm are as follows:

Step 1. Assign the subcarriers to each user in a way that maximizes the overall throughput while maintaining rough proportionality;

Step 2. Assign the total power $Q$ to allocated subcarriers using the multidimension waterfilling algorithm for the bad channel gain user group and the equal power allocation for the good channel gain user group.

\subsection{Subcarrier Allocation by Avoiding Using Ill-Conditioned Subcarrier}

This step allocates the per user assignment of subcarriers $N_{k}$, which is the number of subcarrier for user $k$ and is determined by the average channel gain of each user, in a way that maximizes the overall throughput while maintaining rough proportionality. In this subcarrier allocation algorithm, equal power distribution is assumed across all subcarriers, and we define $\Omega_{k}$ as the set of subcarriers assigned to user k. The proposed algorithm is described below.

a.) Initialization

1) sort the users by average channel gains, suppose we get $\overline{H_{1}} \leq \overline{H_{2}} \leq \ldots \leq \overline{H_{m}} \leq \ldots \leq \overline{H_{K}}$ without loss of generality

2) divide the users into two groups: 
bad channel gain group: user_ $_{-} b=\{1,2, \ldots, m\}$

good channel gain group: user $\_g=\{m+1, m+2, \ldots, K\}$

3) set $R_{k}=0, \Omega_{k}=\varnothing$ for $k=1,2, \ldots, K$ and $A=\{1,2, \ldots, N\}$

b.) For $k=1$ to $m$

1) find $n$ satisfying $\eta_{k, n} \leq \eta_{k, j}$ for $j \in A$, (MMSVR: $\eta_{k, n}=\sigma_{k, n}^{\max } / \sigma_{k, n}^{\min }$ )

2) let $\Omega_{k}=\Omega_{k} \cup n, \mathrm{~N}_{k}=\mathrm{N}_{k}-1, A=A-\{n\}$ and update $R_{k}$ according to (2)

c.) While $|A|>N-\sum_{i=1}^{m} N_{i}$

1) $u s e r_{-} b=\{1,2, \ldots, m\}$

find $k$ satisfying $R_{k} / \gamma_{k} \leq R_{i} / \gamma_{i}$ for all $i, 1 \leq i \leq m$

2) for the found $k$, find $n$ satisfying

find $n$ satisfying $\eta_{k, n} \leq \eta_{k, j}$ for $j \in A$, (MMSVR: $\eta_{k, n}=\sigma_{k, n}^{\max } / \sigma_{k, n}^{\min }$ )

3) for the found $k$ and $n$,

$$
\begin{aligned}
& \text { if } \mathrm{N}_{k}>0 \\
& \text { let } \Omega_{k}=\Omega_{k} \cup n, A=A-\{n\} \\
& N_{k}=N_{k}-1 \text { and update } R_{k} \text { according to (2) } \\
& \text { else } \\
& u s e r_{-} b=u s e r \_b-\{k\}
\end{aligned}
$$

d.) redo step b.) and c.) for the good channel gain group, i.e., for user index from $k=m+1$ to $K$

In the step a.) of the algorithm, the users are divided into the channel gain bad group user $_{-} b$ and the good group user $_{-} g$ according to the average channel gain. And then all the variables are initialized. $R_{k}$ keeps tracks of the throughput for each user and $\mathrm{A}$ is the set of yet unallocated subcarriers.

The step b.) firstly assigns to each user of group $u_{s e r_{-} b} b$ the unallocated subcarrier that has the minimum MMSVR for that user. Note that an inherent advantage is obtained by the bad channel gain group of users that are able to choose their best subcarrier earlier than the other group.

The step c.) proceeds to assign subcarriers to each user of group user_ $b$ according to the greedy policy that the user which needs a subcarrier most in each iteration gets to choose the best subcarrier that has minimum MMSVR for it. The need of a user is determined by the user who has the least throughput divided by its proportional constant. Once the user gets his assignment of $N_{k}$ subcarriers, he can no longer be assigned with any more subcarriers. $|A|$ here denotes the cardinality of the set $A$. 
The step d.) will assign the remaining subcarriers to the good channel gain group. We firstly change the condition "For $k=1$ to $m$ " into "For $k=m+1$ to $K$ " and redo the second step b.). And then we change the condition $|A|>N-\sum_{i=1}^{m} N_{i}$ into $A \neq \varnothing$ and change the selection range $(1 \leq i \leq m)$ into $(m+1 \leq i \leq K)$ so that we can redo the third step c.). Finally all of the subcarriers will be assigned.

By dividing the users into two groups, the users of group user_ $b$ get the inherent advantage to choose their best subcarriers firstly. Thus the power allocated to them in the next step will be deceased significantly compared with other algorithms without giving a priority to bad user group in terms of subcarrier allocation. Accordingly the power allocated to group user $\_g$ will be increased because of the fixed total power in the system. In this way the system throughput can be maximized for the tradeoff between the amount of allocated power and the number and quality of assigned subcarriers for users. Furthermore the proportional fairness will also be roughly guaranteed by step c.).

\subsection{Power Allocation among Users and Subcarriers}

The subcarrier algorithm in step 1 is for each user to use the subcarriers with low MMSVR as much as possible. However, this is not optimum because equal power distribution in all subcarriers is assumed. In this step, we propose a low complexity and efficient power allocation algorithm based on the user grouping criteria in step 1 .

For the bad user group, the power is allocated among the assigned subcarriers for each user by using the multi-dimension water-filling method. In this way, the power efficiency can be improved a lot because of the gain of waterfilling algorithm when SNR is lower. The multi-dimension water-filling method is to find the optimal power allocation as follows.

The power distribution over subcarriers is

$$
q_{n}^{*}=\max \left(0, q_{n}\right)
$$

where $q_{n}$ means the power for subcarrier $n$ and it is the root of the following equation,

$$
\sum_{i=1}^{M_{k_{n} n}} \frac{\lambda_{k_{n} n}^{(i)}}{\lambda_{k_{n} n}^{(i)} q_{n}+N_{0}}+\alpha=0, n=1,2, \ldots, N
$$

where $k_{n}$ is the allocated user index on subcarrier $n ; \alpha$ is the water-filling level which satisfies $\sum_{n=1}^{N^{\prime}} q_{n}^{*}=Q^{\prime}$ where $Q^{\prime}$ and $N^{\prime}$ are the total power for bad user group and the number of subcarriers for bad user group, respectively.

For the good user group, equal power allocation among the assigned subcarriers is used because there is only a little bit difference between equal power allocation and power allocation using water-filling algorithm when the SNR is high. In this way, almost the same system throughput could be achieved and furthermore the complexity could be reduced significantly. 
Once the power allocation for each subcarrier is decided, all of the transmitter antennas use the corresponding power for that subcarrier. Finally, the goal of maximizing the total throughput while maintaining relatively proportional fairness will be achieved after this proposed efficient and low complex power allocation scheme.

\section{Simulation Results and Analysis}

In this section, simulation results are presented to demonstrate the performance of the proposed algorithm. In the simulations, the wireless channel between a couple of transmit antenna and receiver antenna is modeled as a frequency selective channel consisting of six independent Rayleigh multipaths. Each multipath component is modeled by Clarke's flat fading model. The number of users is 4 , and we assume that the average channel gain for user 1 is $10 \mathrm{~dB}$ higher than user2, user3 and user4. The number of antennas is $\mathrm{T}=\mathrm{R}=2$ and each couple of transmit antenna and receiver antenna is independent to the other couples. The total transmit power is $1 \mathrm{~W}$. The total bandwidth $\mathrm{B}$ is $1 \mathrm{MHz}$, which is divided into 64 subcarriers.

In Fig. 2, we show the system throughput of the proposed algorithm in a four-user MIMO-OFDM system vs. different fairness constraints, that is, $\gamma_{1}=2^{m}, \gamma_{2}=\gamma_{3}=\gamma_{4}=1$ and $m$ is from $[0,1,2,3,4,5]$. Thus we have 6 sets of fairness constrains. Fig. 2 also shows the system throughput achieved by the method in [18] which is system throughput maximization scheme, and the system throughput achieved by a static FDMA system, in which each user is allocated with an equal number of subcarrier and it is not changing with the channel variation. The system throughputs in Fig.2 are system throughputs averaged over 5000 channel realizations. It can be seen that the two adaptive resource allocation schemes can achieve a significant throughput gain over the static FDMA.

We also notice that the system throughput maximization method in [18] achieves the maximum system throughput, because all the resources are allocated to the users with the best channel gains. The system throughput achieved by the proposed algorithm varies as the rate constraint changes. As more priority is allocated to user 1 , i.e., as the index $\mathrm{m}$ increases, higher total system throughput is achieved. This is reasonable since user 1 has higher average channel gain and hence can more efficiently utilize the resources. And we can see that the throughput distribution of the method in [18] and static FDMA cannot be changed by varying the gamma set values, because there is no fairness guarantee mechanism in these systems.

Fig. 3 shows the normalized throughput distribution among users for gamma-set index $m=3$ where $\gamma_{1}=8$ and $\gamma_{2}=\gamma_{3}=\gamma_{4}=1$. The normalized throughput for user $\mathrm{k}$ is given by $R_{k} / \sum_{i=1}^{4} R_{i}$. The throughput distributions of four users MIMO OFDM downlink system are shown using the proposed resource allocation algorithm, the throughput maximization scheme in [18] and static FDMA scheme, respectively. It can be seen that the total throughput maximization method in [18] achieves the largest total throughput and user 1 gets most of the subcarriers and occupies most of the total throughput. The throughput for user 2, user 3 and user 4 is very little. Static FDMA tends to allocate similar throughput to each user, since all users get the same number of subcarriers to transmit. However, with the proposed resource allocation algorithm, the throughput is well distributed, very close to the defined ideal rate constraints, among users. 


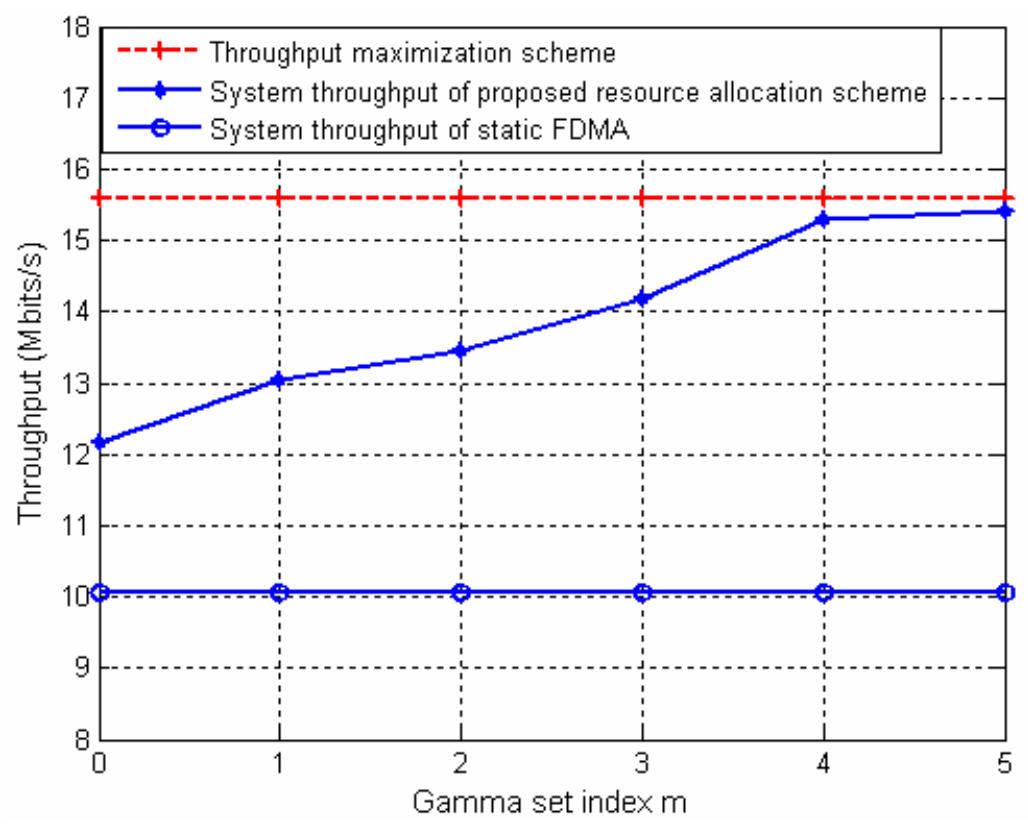

Fig. 2. System throughput of 4 users MIMO-OFDM systems vs. various gamma sets

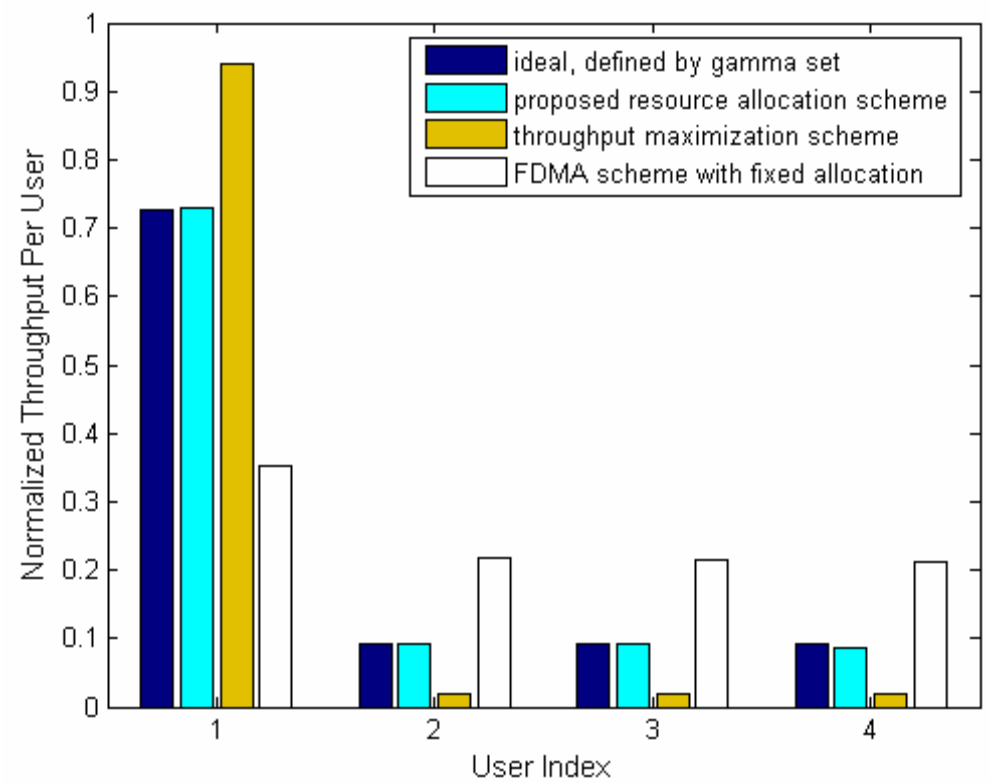

Fig. 3. Normalized throughput ratios distribution among users for the 4 users multiple antenna systems with $\gamma_{1}=8$ and $\gamma_{2}=\gamma_{3}=\gamma_{4}=1$ 


\section{Conclusions}

This paper presents a new method to solve the subcarrier and power allocation problem for the MIMO-OFDMA system. Allocations of subcarrier and power are carried out separately. For the subcarrier allocation, it assigns the subcarriers for each user by dividing the users into groups and also by avoiding using the large MMSVR subcarriers in the second step. The proposed power allocation scheme adopts different algorithms to different user groups in order to achieve high throughput with reduced complexity. Simulation results show that the proposed method can achieve significant throughput gain over the static FDMA. Using the proposed method the system throughput is distributed more fairly among users.

Acknowledgments. This work was supported by Samsung Electronics under the project on 4G wireless communication systems and Yonsei University Institute of TMS Information Technology, a Brain Korea 21 program, Korea.

\section{References}

1. Pualraj, A., Gore, D., Nabar, R., Bolcskei, H.: An overview of MIMO communications - a key to gigabit wireless. Proc. IEEE 92, 198-218 (2004)

2. Goldsmith, A., Jafar, S., Jindal, N., Vishwanath, S.: Capacity limits of MIMO channels. IEEE J. Select. Areas Commun. 21, 684-702 (2003)

3. Stuber, G., Barry, J., McLaughlin, S., Li, Y., Ingram, M., Pratt, T.: Broadband MIMOOFDM wireless communications. Proc. IEEE 92, 271-294 (2004)

4. Van Zelst, A., Schenk, T.: Implementation of a MIMO OFDM-based wireless LAN system. IEEE Trans. Signal Proc. 52, 483-494 (2004)

5. Bjerke, B., Proakis, J.: Multiple-antenna diversity techniques for transmission over fading channels. Proc. IEEE Wireless communication and networking conference 3, 1038-1042 (1999)

6. Gesbert, D., Shafi, M., Shiu, D., Smith, P., Naguib, A.: From theory to practice: an overview of MIMO spacetime coded wireless systems. IEEE J. Select. Areas Commun. 21, 281-302 (2003)

7. Telatar, I.E.: Capacity of multi-antenna Gaussian channels. European Trans. Telecomm. Related Technol. 10, 585-595 (1999)

8. Chiani, M., Win, M.Z., Zanella, A.: The distribution of eigenvalues of a Wishart matrix with correlation and application to MIMO capacity. Proc. of IEEE Globecom conference 4, 1802-1805 (2003)

9. Martin, C., Ottersten, B.: Asymptotic eigenvalue distribution and capacity for MIMO channels under correlated fading. IEEE Trans. Wireless Commun. 3, 1350-1359 (2004)

10. Malik, R.K.: The pseudo-Wishart distribution and its application to MIMO systems. IEEE Trans. Inform. Theory 49, 2761-2769 (2003)

11. Huang, D., Letaief, K.B.: Pre-DFT processing using eigen-analysis for coded OFDM with multiple receive antennas. IEEE Trans. Commun. 52, 2019-2027 (2004)

12. Huang, D., Letaief, K.B.: Symbol based space diversity for coded OFDM systems. IEEE Trans. Wireless Commun. 3, 117-127 (2004) 
13. Wong, C.Y., Cheng, R.S., Letaief, K.B., Murch, R.D.: Multiuser OFDM with adaptive subcarrier, bit, and power allocation. IEEE J. Select. Areas Commun. 17, 1747-1758 (1999)

14. Kivanc, D., Li, G., Liu, H.: Computationally efficient bandwidth allocation and power control for OFDMA. IEEE Trans. Wireless Commun. 2, 1150-1158 (2003)

15. Shen, Z., Andrews, J.C., Evans, B.L.: Adaptive resource allocation in multiuser OFDM systems with proportional rate constraints. IEEE Trans. Wireless Commun. 4, 2726-2737 (2005)

16. Pan, Y.H., Letaief, K.B., Cao, Z.G.: Dynamic spatial subchannel allocation with adaptive beamforming for MIMO/OFDM systems. IEEE Trans. Wireless Commun. 3, 2097-2107 (2004)

17. Rey, F., Lamarca, M., Vazquez, G.: Robust power allocation algorithm for MIMO OFDM system with imperfect CSI. IEEE Trans. Signal Proc. 53, 1070-1085 (2005)

18. Li G., Liu, H.: Capacity analysis on downlink MIMO OFDMA system. Submitted to IEEE Trans. Wireless Commun. (2004)

19. Li, G., Liu, H.: On the optimality of OFDM in multiuser multicarrier MIMO systems. Proc.IEEE Vehicular Technology Conference 4, 2107-2111 (2005) 